\title{
RUPTURA UTERINA
}

\section{A propósito de 34 historias clínicas del Hospital San Juan de Dios, Cúcuta}

\section{Dr. Alberto Duarte Contreras* \\ Dr. Rafael Darío Rolón Duarte*}

\section{I - Introdueción}

La ruptura uterina es el accidente más grave que puede ocurrirle a una mujer embarazada, ya que pone en serio peligro tanto su vida como la del producto; al decir de Thompson es una experiencia traumatizante para la madre y preferencialmente para el médico. Ocurre después de la semana 28 cuando el feto es viable $y$ la mayoría de las veces durante el trabajo de parto. Puede incluir tanto el segmento como el cuerpo uterino de manera separada o en conjunto y abarcar toda la pared muscular o parte de ella.

Su estudio sirve para valorar no solamente el cuidado obstétrico de una institución, sino también el nivel cultural de la comunidad que ella atiende. De aquí que la revisión periódica del tema redunde no sólo en provecho de los centros hospitalarios sino también en pro de la comunidad, ya que el gran número de rupturas producidas fuera de los servicios asistenciales debería despertar el interés de las autoridades sanitarias por la prevención de esta complicación que tan despiadadamente eleva la morbimortalidad materno fetal.

Quisimos conocer el interés de los hospitales de nuestro país sobre este tópico y revisamos la bibliografía nacional a nuestro alcance: en verdad que es muy escasa. (20, 21, 29, 34, $39,42,44,52,54,55)$.

En nuestra revisión seguiremos la siguiente clasificación: (49).

Ruptura uterina $\left\{\begin{array}{l}\text { Espontánea } \\ \text { Traumática } \\ \text { Mal uso de ocitócicos } \\ \text { Abandono del trabajo de parto }\end{array}\right\}$ latrogénica

\section{II - Incidencia}

Sobre un total de 26.111 partos atendidos en el período 1960-1971 encontramos 34 historias de ruptura uterina, lo que nos da una incidencia de 1 por 767 partos. Si tomásemos en cuenta que 17 rupturas se presentaron fuera del servicio, nuestra incidencia depurada sería de 1 por 1.535 .

* Del Departamento de Gineco-Obstetricia del Hospital San Juan de Dios, Cúcuta. Colombia. 
Faro en el presente trabajo incluiremos de manera global todas las rupturas atendidas, producidas dentro o fuera del Hospital, dato que debe tenerse en cuenta para la comparación con otras estadísticas. No encontramos rupturas de repetición. Keifer al revisar 9.302 rupturas observó ruptura de repetición en 27 pacientes $(0,29 \%)(27)$.

CUADRO N: 1

RUPTURA UTERINA

INCIDENCIA COMPARATIVA EN AMERICA LATINA

\begin{tabular}{lrl}
\hline País & Incidencia & Autor \\
\hline Argentina & 1 por 4.273 partos & Nolting (37) \\
Chile & 2.000 & Mansilla (31) \\
Colombia & 1.166 & Ramírez-M. (42) \\
Perú & 1.190 & Jordan (25) \\
México & 1.055 & Castelazo (8) \\
Venezuela & 1.055 & Viso y Agüero (51) \\
Cuba & 1.022 & Marrero (33) \\
Venezuela & 790 & López (30) \\
San Salvador & 728 & Orellana (38) \\
Perú & 694 & González (19) \\
México & 651 & Herrera (23) \\
Paraguay & 515 & Paredes (41) \\
Brasil & 502 & Fernández (13) \\
Bolivia & 348 & Garcés (16) \\
PRESENTE ESTUDIO & 767 & Duarte-C. \\
\hline
\end{tabular}

Revisando la literatura mundial se cncuentran clatos que van desde una ruptura por cada 93 partos hasta una por cada $11.365(1,6,23,24)$, lo que hace muy difícil la comparación de datos estadísticos debido a la diversidad de niveles socio-económicos de la población atendida, a múltiples factores inherentes a la dotación en personal $y$ en elementos y a la presentación, depurada o no, de la casuística. Si dejamos a un lado los centros eminentemente especializados y comparamos nuestras cifras con las de instituciones latinoamericanas de un nivel y funcionamiento similar al nuestro, encontramos que la incidencia de nuestro Hospital es alta.

La ruptura uterina debería ser una contingencia de excepción en centros especializados y en instituciones universitarias, y aún en hospitales generales como el nuestro, de atención gratuita; el índice de frecuencia debería ser más bajo que el registrado en todos los estudios que nosotros conocemos. Algunos autores aceptan que la incidencia en centros especializados se ha estabilizado de tiempo atrás entre una por cada mil a una por cada tres mil partos, y de una por cada 93 a una por cada mil partos en servicios obstétricos de hospitales generales $(6,23)$. Otros como Keifer aseveran que la incidencia va ei aumento progresivo, y así, al hacer una revisión en Washington de los partos atendidos de 1943 a 1962 encuentra que en los primeros diez años la frecuencia es de una ruptura por cada 5.354 partos, en tanto que en la década siguiente es de una ruptura por cada 2.665 partos, lo que representa un aumento en el $50 \%$ (27). Y Palmer y Friedman (40) en un trabajo similar sobre partos atendidos en treinta años confirman el estudio de Keifer. 
En nuestro medio podemos invocar como explicación a este fenómeno de estabilización o de aumento de la incidencia, que sí la hemos observado, los siguientes factores: a) la falta de una consulta prenatal adecuada, b) falta de un servicio social operante, c) el número cada vez mayor de cicatrices uterinas, d) el ningún control en el ejercicio de comadronas, irresponsables en la mayoría de los casos, e) la venta casi libre de ocitócicos en las farmacias, f) el temor a la atención hospitalaria, a más de otros factores de orden socio económico que obstaculizan a las pacientes llegar oportunamente al servicio y aún le impiden asistir a él.

\section{III - Consulta Prenatal}

Ninguna de las pacientes había asistido a consulta prenatal: ello explica claramente por qué 25 habían sido atendidas en sus casas por comadronas antes de acudir al hospital y por qué 17 llegaron con ruptura uterina, cinco de ellas en estado de shock.

Cuando la consulta prenatal vigila la mayoría de las gestantes a quienes más tarde se les atiende el parto, como sucede en los centros especializados, es lógico que disminuya el número de rupturas uterinas ya que muchas de ellas son previsibles y se pueden evitar.

\section{IV - Edad de las pacientes}

CUADRO N: 2

RUPTURA UTERINA

EDAD

\begin{tabular}{ccccc}
\hline Edad & & No & Pacientes & $\%$ \\
\hline 18 & a & 20 & 4 & 11.8 \\
21 & a & 25 & 9 & 26.5 \\
26 & a & 30 & 7 & 20.6 \\
31 & a & 35 & 8 & 23.5 \\
36 & a & 40 & 6 & 17.6 \\
\hline
\end{tabular}

Dos pacientes de 18 y dos de 40 años fueron las de mayor $y$ menor edad en nuestra serie. El grupo comprendido entre 21 y 30 años (46.1\%) es sensiblemente igual al de 31 a 40 años $(41.1 \%)$, cifras éstas similares a las de otros autores. La edad promedio de nuestras pacientes fue de 29 años.

V - Paridad

CUADRO N: 3

RUPTURA UTERINA

PARIDAD

\begin{tabular}{lccr}
\hline Para & $N^{0}$ & Pacientes & $\%$ \\
\hline & & 2 & 5.9 \\
II a VI & 21 & 61.7 \\
VII a XVII & 11 & 32.4 \\
\hline
\end{tabular}

Dos pacientes primigestas de 19 y 21 años respectivamente figuran en nuestra serie; 21 hacen parte del grupo de P. II a P. VI; y once pertenecen a las grandes multíparas. Ello concuerda con las estadísticas por nosotros conocidas $(5,23,31,40$, 42). En ninguno de nuestros casos podemos atribuir la ruptura uterina a la multiparidad exclusivamente pues siempre encontramos algún otro factor determinante.

\section{VI - Antecedentes quirúrgicos}

CUADRO № 4

RUPTURA UTERINA ANTECEDENTE QUIRURGICO

\begin{tabular}{|c|c|c|}
\hline $\begin{array}{l}\text { Antecedente } \\
\text { quirúrgico }\end{array}$ & No Paceintes & $\%$ \\
\hline 1 cesárea & 8 & 23.5 \\
\hline 2 cesáreas & 1 & 2.9 \\
\hline 3 cesáreas & 2 & 5.9 \\
\hline
\end{tabular}

Solamente encontramos la cesárea como antecedente en nuestra casuística, y la encontramos en once pa- 
cientes $(32.35 \%)$, lo que habla muy a las claras sobre el valor etiológico de esta circunstancia, y más aún cuando a ocho se les había practicado solamente una cesárea. Keifer al estudiar 9.302 casos de ruptura uterina recopilados en 705 trabajos de la literatura universal encontró que en el $34 \%$ estuvo presente el antecedente de cesárea (27).

Aun cuando en nuestro hospital se cierra el segmento en dos planos con sutura continua, con mayor frecuencia que con sutura en un plano y puntos separados, en la proporción de 20 a $1(10,14)$, es notorio el hecho de que a todas las pacientes con ruptura uterina y antecedente de cesárea se les había practicado la sutura en dos planos según la técnica de Kerr. Nosotros recalcamos a toda paciente con cicatriz uterina la necesidad de buscar asistencia hospitalaria en su futuro obstétrico.

\section{VII - Presentación}

\section{CUADRO № 5}

RUPTURA UTERINA

PRESENTACION

\begin{tabular}{|c|c|c|c|}
\hline Presentación & $\mathbf{N}^{0}$ & Pacientes & $\%$ \\
\hline Cefálica & & 26 & 76.7 \\
\hline Hombro & & 4 & 11.7 \\
\hline Podálica & & 2 & 5.8 \\
\hline $\begin{array}{l}\text { Flotando en } \\
\text { cavidad abdominal }\end{array}$ & & 2 & 5.8 \\
\hline
\end{tabular}

Cuatro pacientes con presentación de hombro y dos con presentación de pelvis ingresaron con ruptura uterina. Dos fetos muertos llegaron al servicio flotando en cavidad abdomi- nal junto con placenta. Es de notar que la presentación de hombros la encontramos en el $11.7 \%$, cifra ésta muy superior a la aceptada por normal en las estadísticas generales (0.7 a $0.5 \%$ ).

No estamos en capacidad de informar sobre la variedad de posición, ya que este dato no está consignado en todas las historias revisadas.

\section{VIII - Productos}

\section{CUADRO Nํ 6 \\ RUPTURA UTERINA \\ PRODUCTOS}

\begin{tabular}{lcc}
\hline Productos & Número & $\%$ \\
\hline Vivos & 12 & 35,3 \\
Muertos & 22 & 64.7 \\
\hline
\end{tabular}

Las 17 pacientes que llegaron al servicio con ruptura uterina ingresaron con óbito fetal. Cinco muertes fetales ocurrieron dentro del hospital, una de ellas por retención de cabeza última en paciente sin antecedente de cesárea. No tuvimos mortalidad postnatal.

Nuestra cifra de mortalidad perinatal $(64.7 \%)$ está muy cercana a la encontrada en Bogotá por RamírezMerchán $(63.7 \%)$ (42) y muy similar a la de otras instituciones de América, como se observa en el Cuadro 7. Si comparamos este cuadro con el de incidencia (Cuadro No 1), extrañamos el hecho de que estadísticas con cifras de frecuencia de ruptura relativamente bajas, den un tributo tan elevado a la mortalidad perinatal. Si nuestra estadística fuera depurada, la cifra de mortalidad perinatal sería de $29.43 \%$. 
CUADRO N: 7

RUPTURA UTERINA

MORTALIDAD PERINATAL EN INSTITUCIONES DE AMERICA

\begin{tabular}{|c|c|c|}
\hline País & $\%$ & Autor \\
\hline México & 27 & Herrera-Lasso (23) \\
\hline Perú & 38 & Jordan (25) \\
\hline Venezuela & 52.7 & Calcaño (7) \\
\hline USA. ( 14 hospitales) & 62.7 & Colden (18) \\
\hline Colombia & 63.7 & Ramírez-Merchán (42) \\
\hline Venezuela & 69.56 & López-R. (30) \\
\hline Argentina & 75 & Noltin (37) \\
\hline San Salvador & 83.7 & Orellana (38) \\
\hline México & 96.88 & Martínez-Valadez (32) \\
\hline PRESENTE ESTUDIO & 64.7 & Duarte-Contreras \\
\hline
\end{tabular}

\section{IX - Etiología}

De acuerdo a la clasificación ya enunciada, encontramos los siguientes datos:
Encontramos 12 casos de ruptura espontánea y en 6 de ellos había antecedente de cesárea (una cesárea en 5 pacientes y 3 cesáreas en una paciente). Cuatro de estas pacientes fue-

$$
\text { Ruptura uterina }\left\{\begin{array}{l}
\text { Espontánea } \\
\text { Traumática } \\
\text { Uso indebido de ocitócicos } \\
\text { Trabajo abandonado }
\end{array}\right.
$$

ron llevadas a la sala quirúrgica con diagnóstico de inminencia de ruptura, pero al efectuar la laparotomía encontramos la ruptura uterina; creemos o nó haber hecho el diagnóstico oportuno de ruptura, o que ésta se efectuó en el lapso comprendido entre la decisión de practicar cesárea y el comienzo de la intervención. Consideramos estos casos como espontáneos porque tuvieron un trabajo de parto normal, vigilado y sin aplicación de maniobras ni de ocitócicos.

En lo que respecta a ruptura traumática tenemos 2 fórceps de desprendimiento en pacientes $\mathrm{G} 3$ y $\mathrm{G} 6$ respectivamente que llegaron con trabajo prolongado, sufrimiento fetal, en variedad OIA. Los recién naci- $5\left\{\begin{array}{lr}\text { Fórceps } & 2 \\ \text { Kristeller } & 2 \\ \text { Retenciónc abeza última } & 1 \\ & 6 \\ & 11\end{array}\right.$

dos se calificaron con Apgar de 5 y 10 al minuto y 7 a los 5 minutos $y$ tuvieron un peso de 3.600 y 3.000 gr. El diagnóstico en los dos casos se hizo por revisión de cavidad. Creemos, con otros autores $(9,11,26$, $35,43,46,49)$ que el fórceps bajo, correctamente aplicado, no produce lesión alguna uterina; si la técnica es deficiente podría causar desgarros de cuello solamente. Sin embargo, para no ser dogmáticos, podríamos decir que en nuestros casos es difícil precisar si el fórceps fue o no el causante de la ruptura, o lo fue el trabajo prolongado.

La maniobra de Kristeller la hallamos como causante directo en una primigesta de 21 años que dio a luz 
un producto vivo de $3.000 \mathrm{gr}$. con Apgar 7 al minuto y 8 a los 5 minutos, y en una G9 P7 que dio un producto vivo de $3.620 \mathrm{gr}$. con 6 de Apgar al minuto y 8 a los 5 minutos. Somos enemigos de esta maniobra y la proscribimos en nuestra práctica, pues a más de ser causante de hipoxia puede producir estallido uterino; preferimos siempre la aplicación de un fórceps bajo que trae menos riesgo para la paciente y para el feto.

Una paciente G8, de 34 años, con embarazo de 34 semanas, sin antececlente de cesárea, hace una retención de cabeza última y se le extrae con mucha dificultad, después de intentar varias maniobras, un mortinato de $3.100 \mathrm{gr}$. Nunca permitimos el parto vaginal a pacientes con antecedente de cesárea y en presentación de pelvis (50).

En 6 pacientes encontramos el uso indebido de ocitócicos: llegaron todas al servicio con ruptura y en 2 de ellas, al acto quirúrgico, hallamos el óbito en cavidad abdominal flotando junto con placenta. Nos fue imposible conocer la cantidad de unidades aplicadas y sólo sabemos que su administración se hizo por vía intramuscular. Somos muy cautelosos en la aplicación de ocitocina cuando hay antecedente de cicatriz uterina; también recomendamos no emplear la ocitocina en grandes multíparas pues su uso implica un riesgo elevado en la etiología de la ruptura uterina debido a la degeneración progresiva del tejido muscular a tejido fibroso, menos elástico, a medida que aumenta el número de partos. El reciente trabajo de Awais nos da plena razón (4).

Hubo 11 pacientes con trabajo abandonado: 10 llegaron con ruptura y una inició su trabajo en el servicio con antecedente de tres cesá- reas. Nada podemos decir en concreto sobre la duración de estos trabajos de parto pues los datos suministrados por las pacientes que ingresaron con ruptura, o por sus acompañantes, y que fueron consignados en la historia clínica, son imprecisos y se omitieron en la historia de la paciente cuya ruptura se produjo en el servicio.

En unas pacientes se suman varios factores como posibles causantes de ruptura, lo que dificulta precisar su etiología, como en el caso de una G15 de 39 años con antecedente de cesárea, presentación de hombro, trabajo abandonado en su casa y aplicación de ocitocina; nosotros la clasificamos como uso indebido de ocitócicos.

\section{$x$ - Diagnóstico}

El diagnóstico se hizo antes del acto quirúrgico en 18 pacientes, durante el acto quirúrgico en 9 , en el puerperio inmediato por revisión de cavidad en 4, en el puerperio tardío en 1 y por autopsia en 2. Insistimos en la importancia de practicar una cuidadosa revisión de cavidad uterina después de todo parto complicaod o intervenido, y siempre bajo anestesia.

\section{XI - Localización}

La ruptura se localizó en el segmento en 26 pacientes $(76.4 \%)$, en segmento:y cuerpo en $8(23.6 \%)$, tomando con mayor frecuencia el borde izquierdo del cuerpo; todas las rupturas fueron completas; no encontramos ruptura de cara posterior.

\section{XII - Tratamiento}

Se practicaron 10 histerectomías $(29.4 \%)$ : 5 subtotales $(14.7 \%)$ y 5 totales. Las subtotales se realizaron en pacientes de extrema grave- 
dad que no permitían otra cirugía. A $26(64.6 \%)$ se les practicó histerorrafia y a 7 de éstas se les esterilizó (Pomeroy) teniendo en cuenta la edad, la paridad y el estado de los bordes de la ruptura. A una paciente que entró en estado agónico, y a otra, por falla del servicio, no se les practicó tratamiento alguno (se estudiaron luego por necropsia).

Para nosotros, cuando la ruptura es muy grande o cuando los bordes son muy tortuosos, o la intervención es tardía, o la infección es severa, se impone la histerectomía; dejamos entonces un dren transvaginal $y$, en ocasiones, otro a través de la pared abdominal. Pero cada día somos más partidarios de practicar la histerorrafia siempre que sea posible, ya que conserva la función menstrual y la función reproductiva, lo que actúa como estímulo sicológico a la paciente a más de que produce una menor morbimortalidad materna que cuando se efectúa la histerectomía, si bien no todos los autores son partidarios de esta conducta $(2,17,32$, $36,42,45,47)$. Estamos completa- mente convencidos de que la histerorrafia en estos casos no representa un riesgo mayor en el futuro obstétrico de la paciente que el inherente a una cesárea. $(3,12,27,28,45)$. No tenemos en nuestra casuística parto alguno atendido en paciente con antecedente de ruptura uterina.

\section{XIII - Complicaciones}

A una paciente a quien se le practicó histerectomía subtotal se le produjo una fístula uretero-cérvico-vaginal, y a otra con histerectomía total se le produjo una fístula vésico-vaginal; se les solucionó el problema urológico favorablemente con cirugía posterior. Ciertamente es ésta la complicación más frecuente en esta cirugía de urgencia muy azarosa por cierto dado el deplorable estado general de las pacientes y la friabilidad de los tejidos.

El shock se presentó como complicación en 10 pacientes: shock hipovolémico en 8 y shock bacteriémico en 8 ; en 3 encontramos infección de la herida quirúrgica.

\section{CUADRO NN 8}

RUPTURA UTERINA

MORTALIDAD MATERNA EN AMERICA

\begin{tabular}{lll}
\hline País & $\%$ & Autor \\
\hline Perú & 4.76 & Jordan (25) \\
México & 5 & Herrera-Lasso (23) \\
Canadá & 5 & Bachand (5) \\
Estados Unidos & 7.14 & Herbert (22) \\
Colombia & 7.5 & Ramírez-M. (42) \\
Venezuela & 9.3 & Agüero (20) \\
Argentina & 16 & Nolting (3) \\
México & 18 & Castelazo (8) \\
Paraguay & 18 & Paredes (41) \\
San Salvador & 20.3 & Orellana (38) \\
Venezuela & 22.72 & López-R. (30) \\
Perú & 27.46 & González (19) \\
México & 31.24 & Martínez (32) \\
Brasil & 40 & Fernández (13) \\
Panamá & 60 & Sogandares (48) \\
Bolivia & 61 & Garcés (16) \\
Argentina & 61.5 & Frid (15) \\
PRESENTE ESTUDIO & 17.6 & Duarte-C. \\
\hline
\end{tabular}




\section{XIV - Mortalidad materna}

Seis pacientes murieron $(17.6 \%)$ : 4 por shock hipovolémico y 2 por shock bacteriémico, y en una de éstas se comprobó gangrena gaseosa al practicar la necropsia. Cinco de estas pacientes habían llegado con ruptura y en estado preagónico, y su permanencia en el servicio osciló entre una y cuatro horas.

En todas las estadísticas que pudimos consuliar observamos que el más alto porcentaje de mortalidad lo dan las pacientes abandonadas en sus domicilios que llegan luego a los servicios con ruptura y estado de shock. Nuestra cifra de mortalidad materna sin ser depurada, no obstante las deficiencias inherentes al medio, está entre las más bajas de las estadísticas americanas revisadas. Si diéramos cifras depuradas nuestra mortalidad materna sería apenas de 5.88\%.

\section{XV - Resumen}

Se estudian 34 rupturas uterinas del Hospital San Juan de Dios, de Cúcuta, Colombia durante los años 1960-1971 y se encuentra una incidencia no depurada de una por cada 767 partos; la incidencia depurada es de una por 1.535 .

Se comentan y se comparan todos los datos obtenidos con los de otros trabajos y otras latitudes.

Se encuentra el antecedente de cesárea en el $32.35 \%$ de los casos.

La mortalidad materna es del $17.6 \%$ no depurada, y del $5.88 \%$ depurada; la mortalidad perinatal no depurada es $64.7 \%$ y depurada $29.43 \%$.

Se practica histerectomía en 10 pacientes $(29.4 \%)$ : subtotal a 5 y total a 5. A 22 pacientes se les practica histerorrafia $(64.6 \%)$ y a 7 de ellas se les hace además esterilización (técnica de Pomeroy) (20.5\%). Dos pacientes $(6 \%)$ no reciben tratamiento quirúrgico y se les practica luego necropsia.

\section{Summary}

A study of uterine rupture cases is made et the Hospital San Juan de Dios, in Cúcuta, Colombia.

Thirty four ruptures were found during the years 1960-1971. This reveals an incidence of one rupture for 767 deliveres, and depurated one for 1.535 deliveres.

It was faund cesarean antecedent in $32.5 \%$ of cases. The maternal mortality was $17.6 \%$, and depurated $5.88 \%$.

The perinatal mortality was $64.7 \%$ and depurated $29.4 \%$.

Histerectomy was made in 10 patients $(29.4 \%)$, of wich half total histerectomy and half supracervical histerectomy.

Histerorrafia was made in 22 patients $(64.6 \%)$, and in 7 of this, Pomeroy was done.

Two patients didnt reciev any treatment, only necropsy. The dates ar compared with those of other hospitals in Colombia and in Latin America.

\section{XVI - BIBLIOGRAFIA}

1 ADCOK, L. Uterine rupture: experience in Ghana. Obstet. Gynec. 22: 671, 1963.

2 AGUERO, $O$. and KIZER, S. Suture of the uterine rupture. Obstet. Gynec. 31: 806, 1968.

3 AGUERO, O. and KIZER, S. Obstetric prognosis of the repair of uterine rupture. Sur. Gynec. Obstet. 127: 528, 1968.

4 AWAIS, G. M. and LABHERTZ, T. B. Auptured uterus. A complication of oxytocin 
induction and high parity. Obstet. Gynec. 36: $465,1970$.

5 BACHAND, M., SANSOULET, C., PLOUFFE, G., VADEBONCOER, R., GAGNIER, M. et BERNARD, C. Les ruptures utérines a l'Hópital de la Miséricorde de 1950-1965. L'Union Méd. du Canada. 96: 957, 1967.

6 BISSON, C. Canad. M. A. J. 57 : 583, 1947.

7 CALCAÑO, J. y FEO, E. La rotura uterina. Rev. Obst. Gin. Venezuela. 21: 331, 1961.

8 CASTELAZO-AYALA, L. Ruptura del útero grávido. Obst. Gin. Latin. Amer. 16: 1, 1918.

9 CUTIÑO, R., ESPERANZA, R., ALVARADO, A. GUERRERO, C. y SUGAR, J. El fórceps en la paciente con antecedente de cesárea previa. Ginec. Obstet. Méx. 160: 249, 1970.

10 DUARTE-CONTRERAS, A. Doscientas cincuenta cesáreas por los métodos combinados de Sardiñas y Fuad Ferreira. Rev. Colomb. Obs. Gin. 14: 381, 1963.

11 ESPINOSA DE LOS REYES, V., SALDAÑA, R., MARADIEGUE, E., SOLORZANO, H. Y RIVERA, E. Consideraciones sobre 1.500 aplicaciones de fórceps. Ginec. Obstet. Méx. 174: 377,1971

12 FARREL, P. Rupture of the uterus. Am. J. Obstet. Gynec. 97: 332, 1967.

13 FERNANDEZ, M. y FERNANDEZ, O. Roturas do utero. Rev. Ginec. Obstet. (Rio). 102: $467,1958$.

14 FERREIRA, F. Aspectos técnicos de cesárea segmentaria. Maternidade e Infancia. 17: 405, 1958.

15 FRID. Citado por Jordan, M. F.

16 GARCES. Citado por López-R.

17 GARCIA-VALENZUELA, R. Y RUBIO-MADARIAGA, R. El tratamiento conservador de las roturas uterinas. Rev. Esp. Obstet. Ginec. 19: 105, 1960.

18 COLDEN, M. and BETSON, J. R. Obstet. Gynec. 13: 506, 1959.

19 GONZALEZ DEL RIEGO. Citado por Jordan, M. F.

20 GUTIERREZ, J. B. Ruptura uterina. Rev. Méd. Bogotá. 21 : 52, 1958.

21 GUZMAN, F. A propósito de dos casos de ruptura uterina completa durante el parto.
Instantáneas Médicas, Bogotá. 15: 46, 1955.

22 HERBERT, A., CLAIBORN Jr. and SCHELIN, E. Am. J. Obst. Gynec. 95: 99, 1967.

23 HERRERA-LASSO, A. F., VELASCO, R. y RICAUD-ROTHIOT, L. Ruptura uterina. Análisis de 100 casos. Ginec. Obstet. Méx. 139: $597,1968$.

24 JAFFE, R. D. Prolonge uterine rupture. Obstet. Gynec. 25: 126, 1965.

25 JORDAN, M. F. y AQUIJE, H. Ruptura uterina. Mem. III Congreso Peruano Obstet. Ginec. Lima. 1968. Pág. 742.

26 KARCHMER, S. y CHAVEZ, J. Riesgo materno y fetal en cirugía obstétrica. Rev. Colomb. Obst. Gin. 23: 297, 1971.

27 KEIFER, W. Rupture of the uterus. Am. J. Obstet. Gynec. 89: 335, 1964.

28 LALUQUE, P. et TOURNIER-LASSERVE, $\mathrm{CH}$. Les ruptures utérines au cours du travail. Gyn. et Obst. (Paris) 61: 756, 1962.

29 LAVEREDE, L. Breves comentarios a la ruptura uterina. Rev. Méd. Cir. Barranquilla. 12: 13, 1945.

30 LOPEZ-RISSO, N. Rotura uterina en el servicio de maternidad del hospital civil de Maracay. Rev. Obst. Gin. Venezuela. 27 : 645, 1967.

31 MANSILLA. Citado por Jordan. M. F.

32 MARTINEZ-VALADEZ, J. y SUAREZ J. Ruptura uterina. Ginec. Obstet. Méx. 156: 453, 1969.

33 MARRERO. Citado por López-R.

34 MOJICA, C. J. A propósito de un caso de ruptura espontánea de la matriz durante el cmbarazo. Rev. Med. Colomb. 2: 1, 1931.

35 NAVARRETE, O. S. El fórceps en la maternidad Concepción Palacios. Obst. Gin. Venezuela. 29: 15, 1969.

36 NEME, B. Rotura uterina e condutta conservadora iatrogenica. An. Brasil Ginec. 62 : $1,1966$.

37 NOLTING, D. E., RAIMONDI, D., PIOVANO, A., TORRES, A. y BACCHIAZ, B. La ruptura uterina en el Instituto de Maternidad Alberto Peralta Ramos. Buenos Aires. Mem. III Congreso Obst. Gin. Lima, 1968. Pág. 724. 
38 ORELLANA, V. R. Rupturas uterinas en el Hospital de Maternidad de San Salvador. Libro Homenaje al Prof. Luis Castelazo Ayala. Tomo I. Pág. 81, 1970.

39 ORTIZ, S. y NIÑO, A. Un caso de duplici-

dad: ruptura e inversión uterina en una paciente de 30 años. San Gil Médico. 1: 54, 1959.

40 PALERME, G. and FRIEDMAN, E. Rupture of the gravid uterus in the third trimester. Am. J. Obstet. Gynec. 94: 571, 1966.

41 PAREDES. Citado por López-R.

42 RAMIREZ-MERCHAN, R. Y ROJAS, L. Roturas uterinas. Rev. Colomb. Obst. Gin. 20: 365, 1969.

43 RAMIREZ-MERCHAN, R. Concepto actual sobre el fórceps. Rev. Colomb. Obst. Gin. 12: 442, 1961.

44 RESTREPO, A. Ruptura espontánea del útero. Rev. Clin. Medellín. 2: 231, 1917.

45 REYES-CEJA, L., CABRERA, R., INFRAN, E. and HERRERA-LASSO, F. Pregnancy following previous uterine rupture. Obstet. Gynec. 34: 387, 1969.

46 RUIZ-VELASCO, V. Pronóstico de la cicatriz post cesárea. Ginec. Obstet. Méx. 20: 1071, 1965.
47 SEPTIEN, J. M. y ROMERO, I. Cambio en el tratamiento de la ruptura uterina. Ginec. Obstet. Méx. 157: 581, 1969.

48 SOGANDARES. Citado por Jordan, M. F.

49 SUAREZ-COBO, M. Comentario al trabajo "Ruptura uterina. Análisis de 100 casos.". Ginec. Obstet. Méx. 139: 607, 1968.

50 TEPPA- T. P. A. Ruptura uterina en podálica. Revisión de 26 años de la maternidad Concepción Palacios. Rev. Obst. Gin. Venezuela. 26: 271, 1966.

51 VISO, R. y AGUERO, O. Rotura uterina. Obstet. Ginec. Latin. Amer. 14: 177, 1956.

52 VEGA, J. A. Ruptura del útero durante el trabajo de parto. Casos vistos en el hospital Monfort de Villavicencio. Rev. Colomb. Obst. Gin. 16: 159, 1965.

53 ZELLER, R., SCHWARTZ, R. and EMIEH, J. Uterine rupture: an analysis of 43 cases. Obstet. Gynec. 27: 859, 1966.

54 Ruptura uterina. Anlisis de los casos presentados en la Clínica de Maternidad de Medellín desde su fundación, marzo 1 de 1954 hasta agosto de 1963 inclusive. Bol. Científico Clin. de Maternidad "Luz Castro de Gutiérrez", Medellín, 6: 13, 1963.

55 Un caso de "parto" a través de la pared abdominal. Bol. Científico Clin. Maternidad "Luz Castro de Gutiérrez". Medellín. 7: 19, 1964. 